\title{
Exploring Hierarchical Rule Systems in Parallel Coordinates
}

\author{
Thomas R. Gabriel, A. Simona Pintilie, and Michael R. Berthold \\ ALTANA Chair for Bioinformatics and Information Mining, \\ Department of Computer and Information Science, \\ Konstanz University, Box M 712, 78457 Konstanz, Germany \\ \{gabriel, pintilie, berthold\}@inf.uni-konstanz.de
}

\begin{abstract}
Rule systems have failed to attract much interest in large data analysis problems because they tend to be too simplistic to be useful or consist of too many rules for human interpretation. We recently presented a method that constructs a hierarchical rule system, with only a small number of rules at each level of the hierarchy. Lower levels in this hierarchy focus on outliers or areas of the feature space where only weak evidence for a rule was found in the data. Rules further up, at higher levels of the hierarchy, describe increasingly general and strongly supported aspects of the data. In this paper we show how a connected set of parallel coordinate displays can be used to visually explore this hierarchy of rule systems and allows an intuitive mechanism to zoom in and out of the underlying model.
\end{abstract}

\section{Introduction}

Extracting rule models from data is not a new area of research. In [1] and [2], to name just two examples, algorithms were described that construct hyperrectangles in feature space. The resulting set of rules encapsulates regions in feature space that contain patterns of the same class. Other approaches, which construct fuzzy rules instead of crisp rules, were presented, for example, in $[3,4,5]$ and $[6]$. What all of these approaches have in common is that they tend to build very complex rule systems for large data sets originating from a complicated underlying system. In addition, high-dimensional feature spaces result in complex rules relying on many attributes and increase the number of required rules to cover the solution space even further. An approach that aims to reduce the number of constraints on each rule individually was recently presented in [7]. The generated fuzzy rules only constrain few of the available attributes and hence remain readable even in the case of high-dimensional spaces. However, this algorithm also tends to produce many rules for large, complicated data sets.

In [8] we described a method that attempts to tackle this inherent problem of interpretability in large rule models. We achieve this by constructing a hierarchy of rules with varying degrees of complexity. The method builds a rule hierarchy for a given data set. The rules are arranged in a hierarchy of different 
levels of precision; each rule only depends on few, relevant attributes thus making this approach also feasible for high-dimensional feature spaces. Lower levels of the hierarchy describe regions in input space with low evidence in the given data, whereas rules at higher levels describe more strongly supported concepts of the underlying data. The method is based on the fuzzy rule learning algorithm mentioned above $[7,9]$, which builds a single layer of rules autonomously. We recursively use the resulting rule system to determine rules of low relevance, which are then used as a filter for the next training phase. The result is a hierarchy of rule systems with the desired properties of simplicity and interpretability on each level of the resulting rule hierarchy. Experimental results demonstrated that fuzzy models at higher hierarchical levels indeed show a dramatic decrease in number of rules while still achieving better or similar generalization performance than the fuzzy rule system generated by the original, non-hierarchical algorithm.

In this paper we show how an accompanying system of inter-connected rule visualizations in parallel coordinates can be used to intuitively explore the rule systems at each level of granularity while at the same time enabling the user to easily zoom in and out of the model, effectively changing to other levels of the hierarchy while maintaining the focus of analysis. The approach is based on recent work on visualization of fuzzy rules in parallel coordinates [10] and extends it using ideas from the information visualization community, so-called structure-based brushing techniques [11]. This method, however, cannot be used intuitively and hence is only useful for an expert user. Here we go beyond solely tying points in each view together, by allowing elements that are connected across different levels of abstraction to be highlighted, i. e. hierarchy layers in the case discussed here. The ability to highlight rule(s) in one layer of the hierarchy and immediately see related rules is a powerful way to quickly increase or reduce the level of detail in an inuitive manner.

The paper is organized as follows: In the next section we briefly describe the used hierarchical rule learning method, followed by an introduction to parallel coordinates, and how normal rule systems can be visualized in the section thereafter. We then describe how hierarchies of rules can be explored in parallel coordinates and illustrate the proposed method using the Iris data set, before we show how larger hierarchical rule sets can be visualized and explored for a number of real world data sets.

\section{Hierarchical Rule System Formation}

The rule induction algorithm used here is based on a method described in [7], which builds on an iterative algorithm. During each learning epoch, i. e. presentation of all training patterns, new fuzzy rules are introduced when necessary and existing ones are adjusted whenever a conflict occurs. For each pattern three main steps are executed. Firstly, if a new training pattern lies inside the supportregion of an existing fuzzy rule of the correct class, its core-region is extended in order to cover the new pattern. Secondly, if the new pattern is not yet covered, 
a new fuzzy rule of the correct class is introduced. The new example is assigned to its core, whereas the support-region is initialized "infinite", that is, the new fuzzy rule covers the entire domain. Lastly, if a new pattern is incorrectly covered by an existing fuzzy rule, the fuzzy points' support-region is reduced so that the conflict is avoided. This heuristic for conflict avoidance aims to minimize the loss in volume. In [9], three different heuristics to determine the loss in volume were compared in more detail. As discussed in [7], the algorithm terminates after only a few iterations over the set of example patterns. The resulting set of fuzzy rules can then be used to classify new patterns by computing the overall degree of membership for each class. The accumulated membership degrees over all input dimensions and across multiple rules are calculated using fuzzy t-norm and t-conorm respectively. For the purpose of this paper, we concentrate on the rules' core only, that is, we consider only the part of each rule where the degree of membership is equal to 1 - resulting in crisp rules ${ }^{1}$.

In [8], an extension of this algorithm was proposed that allows the generation of an entire hierarchy of such rules. The rule layers are arranged in a hierarchy of different levels of precision. Lower levels of the hierarchy describe regions in input space with low evidence in the given data, whereas rules at higher levels describe more strongly supported concepts of the underlying data. We recursively use the above-mentioned classical fuzzy rule induction algorithm to determine rules of low relevance, which are then used as a filter for the next training phase. Training examples that resulted in creation of small, less important rules are therefore excluded from the training phase of the next layer, resulting in a more general rule system, ignoring the withheld, small details in the training data. The result is the desired hierarchy of rule systems with an increasing generality towards higher levels. In [9] it was shown that the accuracy of these hierarchies is comparable to the non-hierarchical algorithm. Additionally, it was shown that the general rule system towards the top of the hierarchy alone often also show comparable performance, sometimes even outperforming the classical non-hierarchical system.

\section{Rule Systems in Parallel Coordinates}

Parallel coordinates $[12,13]$ allow $n$-dimensional data to be visualized in $2 \mathrm{D}$ by transforming multi-dimensional problems into 2D patterns without loss of information. Visualization is facilitated by viewing the $2 \mathrm{D}$ representation of the $n$-dimensional data. Each of the $n$ coordinate axes is taken and lined up in parallel, resulting in the basis for parallel coordinates. The distance between each adjacent axis is assumed to be equal to 1 . A point in $n$-dimensional space becomes a series of $n-1$ connected lines in parallel coordinates that intersect each axis at the appropriate value for that dimension. A parallel coordinates example

1 Obviously, the extensions for visulizations of fuzzy rules described in [10] can also be used but as this is not the central focus of this paper, it has therefore been omitted for reasons of space. 

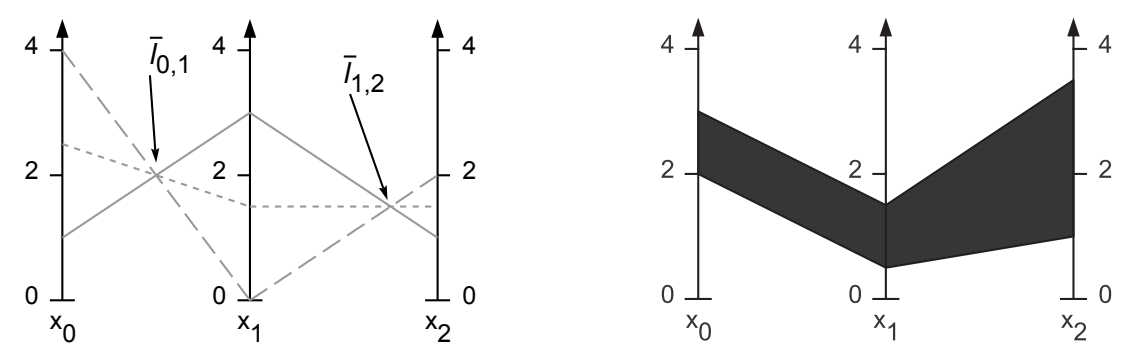

Fig. 1. Left: A parallel coordinate depiction of 3 points on a line in 3D. Right: A rule in parallel coordinates, expressing a disjunctive constraint on all three features.

of 3 points in $3 \mathrm{D}, \boldsymbol{a}=(1,3,1), \boldsymbol{b}=(4,0,2)$, and $\boldsymbol{c}=(2.5,1.5,1.5)$, from a line is shown in Figure 1.

The dual of an $n$-dimensional line in Cartesian coordinates is a set of $n-1$ points in parallel coordinates [14,15]. For the example in Figure 1 (left), these are indicated by $\bar{l}_{0,1}=(0.5,2)$ and $\bar{l}_{1,2}=(0.75,1.5)$, which uniquely describe a line in 3 dimensions.

The $n$-dimensional line in Cartesian coordinates can be represented by $(n-1)$ linearly-independent equations each of which results from equating a different pair of the following fractions [12]:

$$
\frac{x_{0}-a_{0}}{u_{0}}=\frac{x_{1}-a_{1}}{u_{1}}=\ldots=\frac{x_{n-1}-a_{n-1}}{u_{n-1}} .
$$

Now it may be assumed that the $n-1$ linearly independent equations are obtained from pairing the $n-1$ adjacent fractions, with no loss in generality. This yields

$$
x_{i+1}=m_{i} x_{i}+b_{i}, i=0,1, \ldots, n-2,
$$

where $m_{i}=u_{i+1} / u_{i}$ represents the slope and $b_{i}=\left(a_{i+1}-m_{i} a_{i}\right)$ the intercept of the $x_{i+1}$-axis of the projected line on the $x_{i} / x_{i+1}$-plane. The dual point of the $n$-dimensional line in parallel coordinates therefore corresponds to the set of $n-1$ indexed points:

$$
\left(\frac{i}{1-m_{i}}, \frac{b_{i}}{1-m_{i}}\right), \text { for } i=0,1, \ldots, n-2 .
$$

In [16], an extension of parallel coordinates was presented that allows not only points to be visualized but also crisp and fuzzy rules. Crisp rules result in "bands" going through the parallel coordinates, visualizing the intervals representing the constraints on each axes. In Figure 1 (right) an example in 3D is shown, depicting the rule:

$$
\text { IF } x_{0} \in[2,3] \wedge x_{1} \in[0.5,1.5] \wedge x_{2} \in[1,3.5] \ldots
$$

The inherent imprecision of fuzzy rules was depicted using degrees of shading to visualize the degree of membership at each level, however, for the purpose of this paper we concentrate on crisp rules. The extension to the fuzzy case is straightforward. We will see examples of such visualizations in the next section. 


\section{Exploring Hierarchical Rule Systems: An Example}

To illustrate the proposed hierarchical rule visualization scheme, the well-known Iris data [17] was used. The Iris data consists of 150 four-dimensional patterns describing three classes of Iris plants: Iris-setosa, Iris-versicolor, and Iris-virginica. The four dimensions consist of measurements for the petal and sepal, length and width.

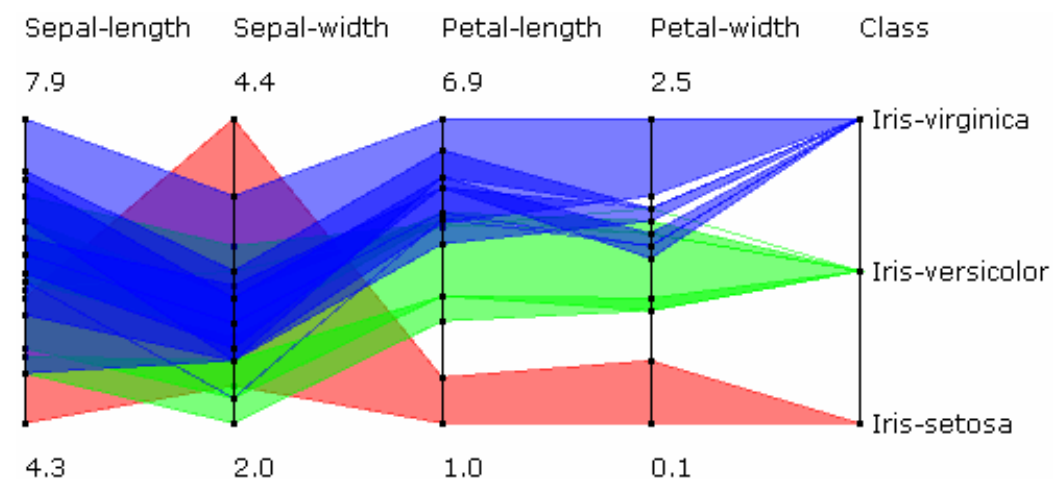

Fig. 2. The flat rule set for the Iris data

Figure 2 shows the flat non-hierarchical rule system as it would be generated by the original rule induction algorithm described in [7]. In Figure 3, the hierarchical rule learner produced three levels of rule systems. The top level has three rules, one for each class, which nicely describe the general trend in the data. At subsequent, lower levels, the granularity increases and finer details of the data are visually depicted. One can clearly see, how four isolated patterns of two classes were filtered out during the first stage of the hierarchy induction.

Obviously, such an easy example is only suitable to demonstrate the algorithm's operation. In the following section, we show how it also works on two real world data sets, discovering interesting structures in the data.

\section{Application to a Real World Problem}

\subsection{Ocean Satellite Images}

The first data set stems from a satellite used primarily to examine the ocean. The images are from the Coastal Zone Color Scanner (CZCS) and are of the West Florida shelf $[18,19]$. The CZCS was a scanning radiometer aboard the Nimbus-7 satellite, which viewed the ocean in six co-registered spectral bands $443,520,550,670,750 \mathrm{~nm}$, and a thermal IR band. It operated from 1979-1986.

The features used were the 443, 520, 550, $670 \mathrm{~nm}$ bands; the pigment concentration value was derived from the lowest 3 bands. Atmospheric correction was 

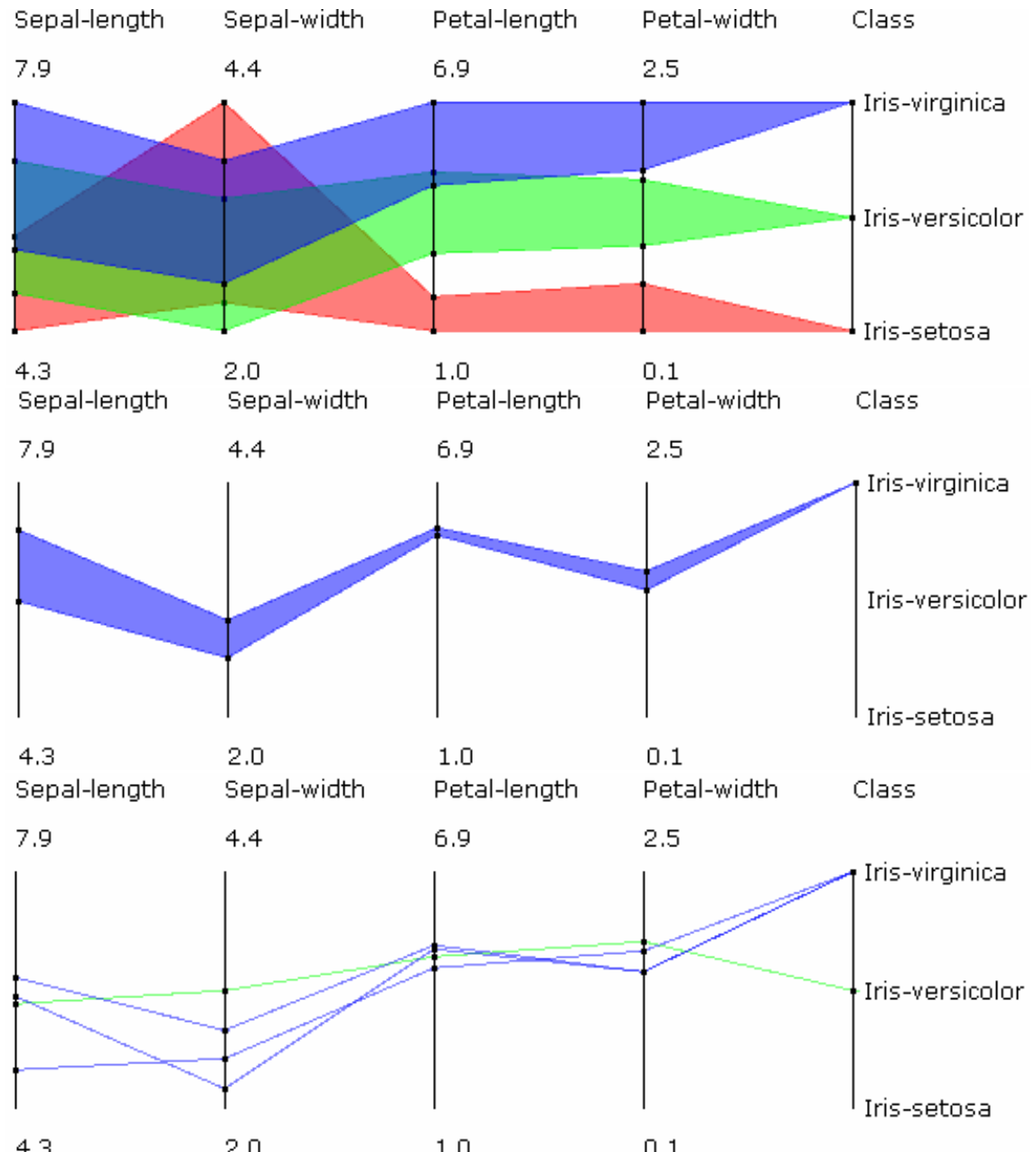

4.3

2.0

1.0

0.1

Fig. 3. The 3-level hierarchy for the Iris data. Bottom: the lowest level, showing rules for four isolated patterns which are in conflict with some of the rules of the higher levels. Middle: the next level, here only containing one rule for class Iris-virginica. Top: the top level, showing the three most general rules, one for each class.

applied to each image [20] before the features were extracted. A fast fuzzy clustering algorithm, mrFCM [21], was applied to obtain 12 clusters per image. There were five regions of interest in each image. These consist of red tide, green river, other phytoplankton blooms, case I (deep) water and case II (shallow) water. Twenty-five images were ground-truthed by oceanographers [22] and eighteen of these were used for training. The eighteen training images were clustered into 12 classes. Each class or cluster was labeled by the ground truth image as its majority class. 

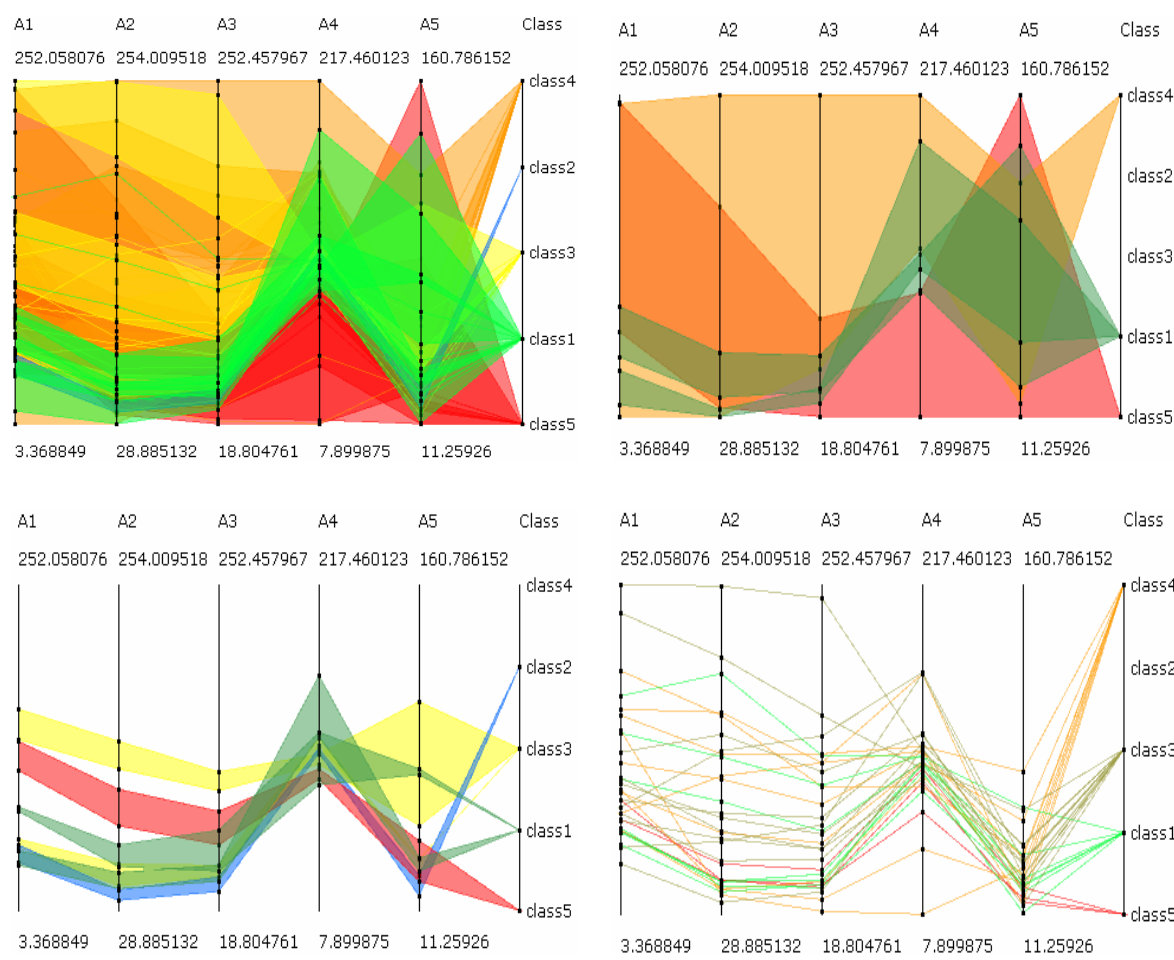

$\begin{array}{lllll}3.368849 & 28.885132 & 18.804761 & 7.899875 & 11.25926\end{array}$

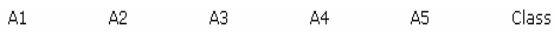

$252,058076254.009518 \quad 252.457967 \quad 217.460123 \quad 160.786152$

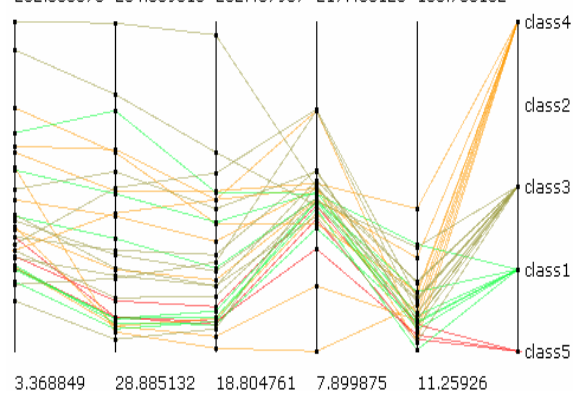

Fig. 4. Top left shows the flat rule system for the ocean satellite image data followed by the 3-level hierarchical rule system. Top right: the highest layer of the hierarchy showing four rules for three of the 5 classes. Bottom left: the middle layer, modeling less important classes and trends. Bottom right: the bottom layer of the hierarchy, modeling outliers, and rare cases.

The labeled cluster centers from the training images were then given to the rule induction tool used also with the Iris data. It generated a set of fuzzy rules, which are shown in Figure 4 (top left). Note how the parallel coordinate display is completely overloaded and essentially useless.

The hierarchical rule induction method generates three layers of hierarchy, which are shown again in Figure 4. Note how the top layer displays only the four most important rules, and even skips rules for two less frequent classes. Those classes are modeled by two extra rules on the middle layer of the hierarchy, which again only displays a few rules (five in this case), hence allowing interpretability even at this level. The bottom layer finally shows rules modeling outliers in the data, indicated by lines. Even this layer of the rule hierarchy still provides an interpretable overview of the structure of the remaining data. It is interesting to note that the complete hierarchy contains less rules than the original flat model itself. Therefore, not only does the hierarchical representation allow better interpretation of the resulting rule models, it is also a more compact representation of the data itself. 


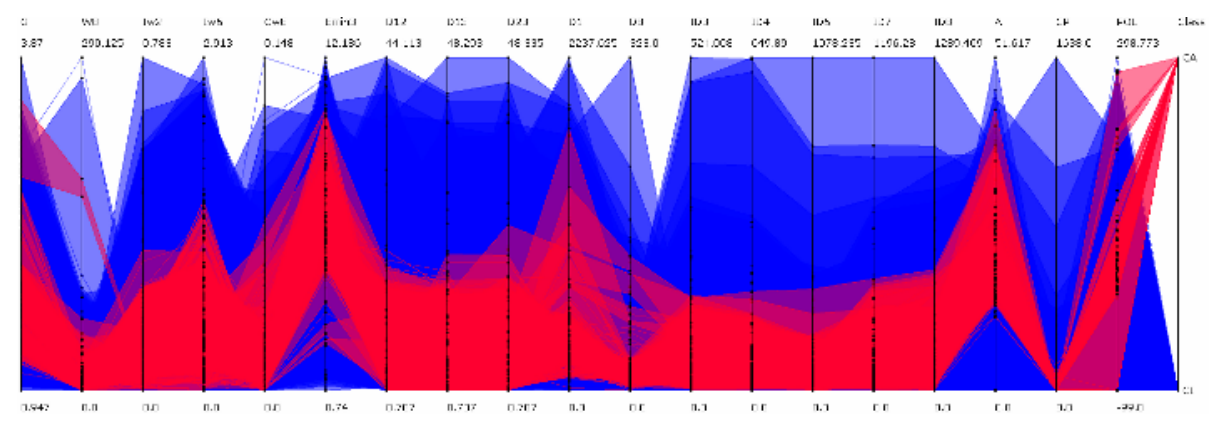

Fig. 5. The bottom layer of the rule hierarchy for NCI's HIV dataset using VolSurf features

\subsection{NCI's HIV Data}

The proposed hierarchical visualization method was also applied to a well-known data set from the National Cancer Institute, the DTP AIDS Antiviral Screen data set [23]. The class assignment, provided with the data, lists compounds that provided at least 50\% protection against HIV on retest as moderately active (CM), compounds that reproducibly provided $100 \%$ protection were listed as confirmed active (CA), and compounds not meeting these criteria were listed as confirmed inactive $(\mathbf{C I})$. Available online ${ }^{2}$ are screening results and chemical structural data on compounds that are not covered by a confidentiality agreement. We have generated VolSurf descriptors for these compounds [24], resulting in

- 325 compounds of class CA,

- 877 compounds of class $\mathbf{C M}$, and

- 34, 881 compounds of class CI.

VolSurf computes 2D molecular descriptors based on grid maps modeling interaction energies at a molecular level. The used distance metric was the usual Euclidean distance, computed on a subset of 15 of the available descriptors. Patterns of class CM were not used in the following experiments.

Figure 5 shows the bottom level of the resulting hierarchy of rule models. Due to heavy overlap of many rules (210 in this case), not much useful information can be derived from this picture.

Figure 6 shows the top level of the model consisting of 30 rules $(7$ for class CA). Note how, especially for class CA, which is the class of interest in this application, a number of interesting observations can be made ${ }^{3}$. For instance, two main clusters can be distinguished that are clearly divided along dimensions G, D1, ID7, ID8, and POL. Also, a correlation across several attributes for rules of class CA is visible: ID7, ID8, and D1. In addition, it is interesting to note that

2 http://dtp.nci.nih.gov/docs/aids/aids_data.html

${ }^{3}$ Naturally, the results can be seen more clearly on the screen. 


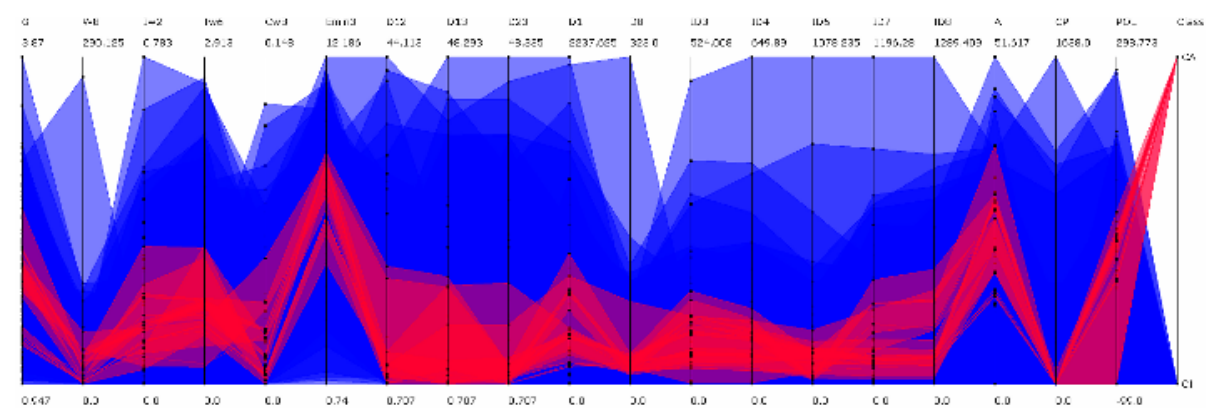

Fig. 6. The top layer of the rule hierarchy for NCI's HIV dataset, containing 30 rules of which only 7 belong to class $\mathbf{C A}$, the class of interest

along attribute Iw2 only rules of class $\mathbf{C A}$ occupy a middle area, where no rules of class $\mathbf{C I}$ interfere.

Since these were early experiments, extensive evaluation with expert feedback was not able to be conducted. It would be interesting to find out if any of the above observations are correlated to information contained in the VolSurf descriptors.

\section{Interactive Rule Exploration and Zooming}

To demonstrate the power of interactive, visual brushing across different views in the parallel coordinate hierarchy, we trained a three-level fuzzy rule hierarchy on the vehicle silhouette dataset from the European StatLog-Project [25]. This 18-dimensional dataset consists of 846 samples belonging to 4 classes. The three levels of the fuzzy rule hierarchy contain 21 rules in the top, 47 in the middle, and 256 at the bottom-most level. Figure 7, 8, and 9 show two of three hierarchy models in parallel coordinates demonstrating the highlighting property of the views. The first picture displays all 256 rules of the bottom level - clearly no exploration is possible. Selecting one of the rules in the top level is shown in Figure 8 , here all other non-selected rules are faded and moved to the background. This selection is automatically propagated to the other layers and highlights related rules in these views. As can be seen in Figure 9, in the bottom layer only 16 rules are related and hence highlighted. The user can easily identify these small rules, which explain outliers or artifacts in the data that are related to the rules selected in the top layer.

\section{Conclusions}

We have presented an approach to visualize hierarchical rule systems using a series of parallel coordinate displays. Experiments on three real world data sets show how complicated rule systems, which would otherwise be uninterpretable in a visual display, show interesting insights when displayed at different levels of 


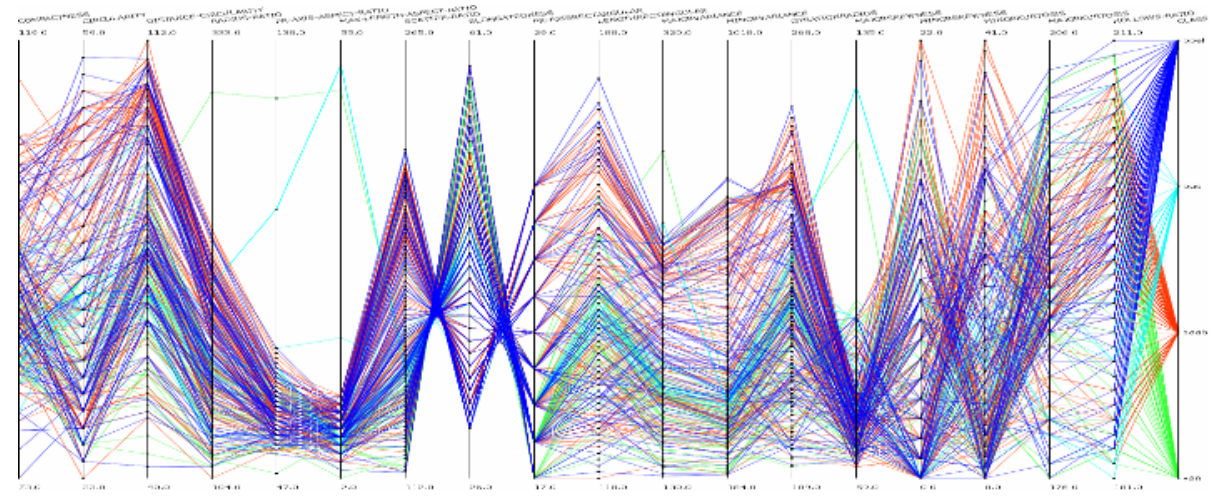

Fig. 7. Bottom level of the three-level hierarchy, which shows 256 rules for 4 classes

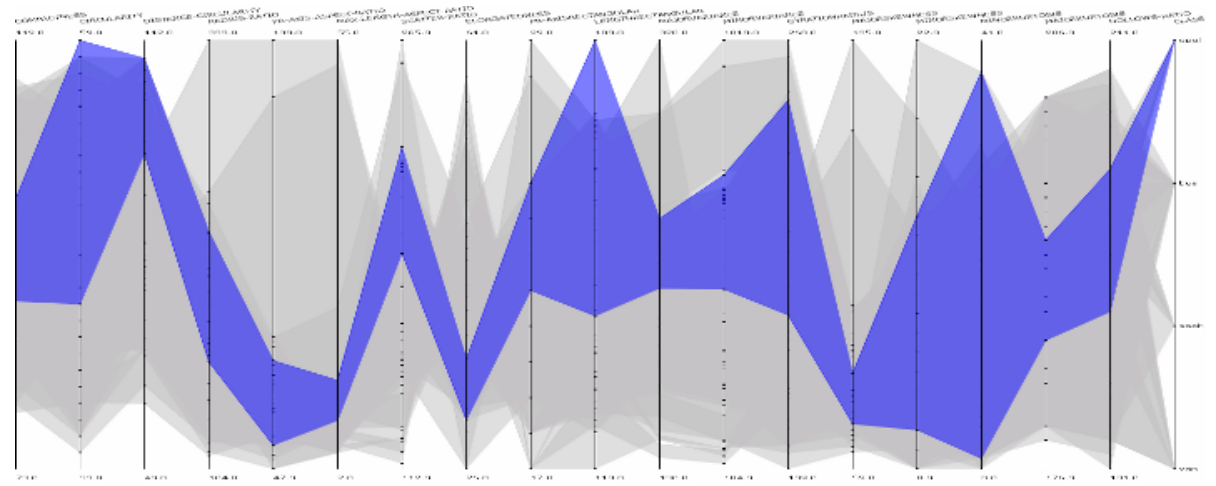

Fig. 8. Top level of the hierarchy, where one rule has been selected and the other 20 rules are faded

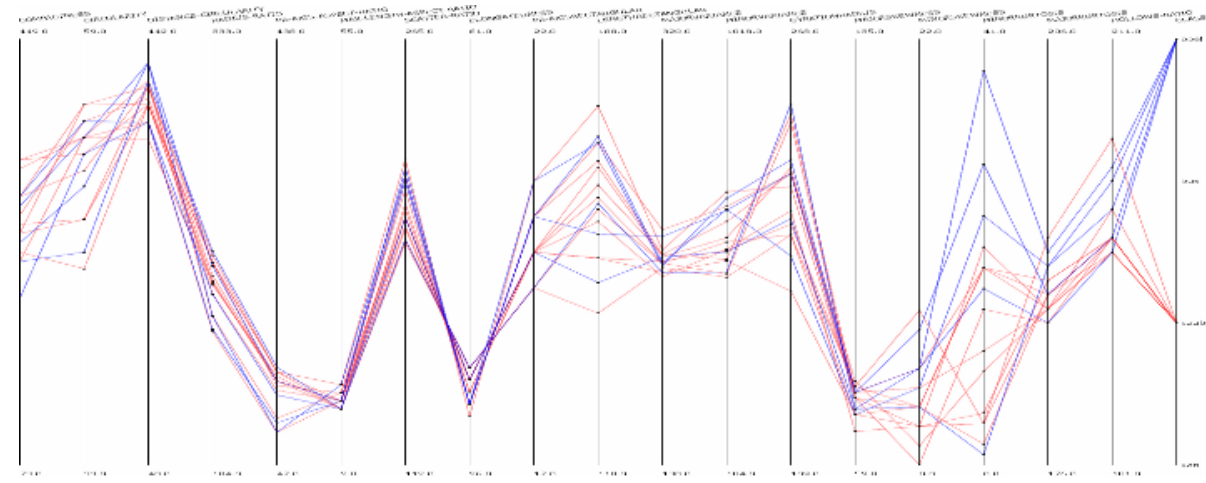

Fig. 9. Again the bottom level, showing 16 of the 256 rules that are related with the rule selected in the top level 
abstraction. The ability to interact with the hierarchical rule system at different levels of detail shows promise for the analysis of large, complicated data sets. We are currently working on extending this tool to allow real visual zooming operations within the same view, which will make this type of hierarchical rule system visualization even more powerful for truly exploratory information mining.

\section{Acknowledgments}

This work was supported by the DFG Research Training Group GK-1042 "Explorative Analysis and Visualization of Large Information Spaces".

\section{References}

1. Salzberg, S.: A nearest hyperrectangle learning method. In: Machine Learning. 6 (1991) 251-276

2. Wettschereck, D.: A hybrid nearest-neighbour and nearest-hyperrectangle learning algorithm. In: Proceedings of the European Conference on Machine Learning. (1994) 323-335

3. Abe, S., Lan, M.S.: A method for fuzzy rules extraction directly from numerical data and its application to pattern classification. IEEE Transactions on Fuzzy Systems 3 (1995) 18-28

4. Higgins, C.M., Goodman, R.M.: Learning fuzzy rule-based neural networks for control. In: Advances in Neural Information Processing Systems. 5, California, Morgan Kaufmann (1993) 350-357

5. Simpson, P.K.: Fuzzy min-max neural networks - part 1: Classification. IEEE Transactions on Neural Networks 3 (1992) 776-786

6. Wang, L.X., Mendel, J.M.: Generating fuzzy rules by learning from examples. IEEE Transactions on Systems, Man, and Cybernetics 22 (1992) 1313-1427

7. Berthold, M.R.: Mixed fuzzy rule formation. International Journal of Approximate Reasoning (IJAR) 32 (2003) 67-84

8. Gabriel, T.R., Berthold, M.R.: Constructing hierarchical rule systems. In Berthold, M.R., Lenz, H.J., Bradley, E., Kruse, R., Borgelt, C., eds.: Proc. 5th International Symposium on Intelligent Data Analysis (IDA 2003). Lecture Notes in Computer Science (LNCS), Springer Verlag (2003) 76-87

9. Gabriel, T.R., Berthold, M.R.: Influence of fuzzy norms and other heuristics on "mixed fuzzy rule formation". International Journal of Approximate Reasoning (IJAR) 35 (2004) 195-202

10. Berthold, M.R., Hall, L.O.: Visualizing fuzzy points in parallel coordinates. IEEE Transactions on Fuzzy Systems 11 (2003) 369-374

11. Fua, Y.H., Ward, M., Rundensteiner, E.A.: Hierarchical parallel coordinates for exploration of large datasets. In: IEEE Conference on Visualization. (1999) 43-50

12. Inselberg, A., Dimsdale, B.: Multidimensional lines I: representation. SIAM J. Applied Math 54 (1994) 559-577

13. Inselberg, A., Dimsdale, B.: Multidimensional lines II: proximity and applications. SIAM J. Applied Math 54 (1994) 578-596

14. Inselberg, A.: Multidimensional detective. In: IEEE Symposium on Information Visualization, InfoVis, IEEE Press (1997) 100-107 
15. Chou, S.Y., Lin, S.W., Yeh, C.S.: Cluster identification with parallel coordinates. Pattern Recognition Letters 20 (1999) 565-572

16. Berthold, M., Hand, D.J., eds.: Intelligent Data Analysis: An Introduction. 2nd edn. Springer Verlag (2003)

17. Fisher, R.A.: The use of multiple measurements in taxonomic problems. In: Annual Eugenics, II. 7, John Wiley, NY (1950) 179-188

18. Zhang, M., Hall, L., Goldgof, D.: Knowledge-based classification of czcs images and monitoring of red tides off the west florida shelf. In: The $13^{\text {th }}$ International Conference on Pattern Recognition. Volume B. (1996) 452-456

19. Zhang, M., Hall, L.O., Goldgof, D.B., Muller-Karger, F.E.: Fuzzy analysis of satellite images to find phytoplankton blooms. In: IEEE International Conference on Systems Man and Cybernetics. (1997)

20. Gordon, H.R., Clark, D.K., Mueller, J.L., Hovis, W.A.: Phytoplankton pigments derived from the nimbus-7 czcs: comparisons with surface measurements. Science 210 (1980) 63-66

21. Cheng, T.W., Goldgof, D.B., Hall, L.: Fast fuzzy clustering. Fuzzy Sets and Systems 93 (1998) 49-56

22. Zhang, M., Hall, L., Goldgof, D.: Knowledge guided classification of coastal zone color images off the west florida shelf. Technical Report ISL-99-11, University of South Florida, Dept. of CSE, USF, Tampa, FL. (1999) Under review in International Journal of Pattern Recognition and AI.

23. Weislow, O., Kiser, R., Fine, D., Bader, J., Shoemaker, R., Boyd, M.: New soluble formazan assay for HIV-1 cytopathic effects: application to high flux screening of synthetic and natural products for AIDS antiviral activity. Journal National Cancer Institute 81 (1989) 577-586

24. Cruciani, G., Crivori, P., Carrupt, P.A., Testa, B.: Molecular fields in quantitative structure-permeation relationships: the VolSurf approach. Journal of Molecular Structure 503 (2000) 17-30

25. Michie, D., Spiegelhalter, D.J., Taylor, C.C., eds.: Machine Learning, Neural and Statistical Classification. Ellis Horwood Limited (1994) 